\title{
ON SOLUTIONS OF ELLIPTIC EQUATIONS THAT DECAY RAPIDLY ON PATHS
}

\author{
D. H. ARMITAGE
}

(Communicated by Barbara Lee Keyfitz)

\begin{abstract}
Let $P(D)$ be an elliptic differential operator on $\mathbb{R}^{n}$ with constant coefficients. It is known that if $u$ is a solution of $P(D) u=0$ on an unbounded domain and if $u$ decays uniformly and sufficiently rapidly, then $u=0$. In this note it is shown that the same conclusion holds if $u$ decays rapidly, but not a priori uniformly, on a sufficiently large set of unbounded paths.
\end{abstract}

Throughout this note $\Omega$ is an unbounded domain in $\mathbb{R}^{n}$, where $n \geq 2$, and $P(D)=\sum_{|\alpha| \leq d} a_{\alpha} D^{\alpha}$ is an elliptic linear differential operator on $\mathbb{R}^{n}$ with constant complex coefficients. In response to a problem proposed for the harmonic case $(P(D)=\Delta$, the Laplacian operator) at a Durham Conference in 1983 [3, Problem 3.27], Armitage, Bagby and Gauthier [1] gave two proofs of the following result.

Theorem A. There exists a continuous function $\varepsilon:[0,+\infty) \rightarrow(0,1]$ with the following property. If $u$ is a solution of $P(D) u=0$ on $\Omega$ such that $|u(x)| \leq$ $\varepsilon(\|x\|)$ for all $x \in \Omega$, then $u=0$.

Some theorems for special domains $\Omega$ in the harmonic and holomorphic ( $n=2$ and $P(D)=\bar{\partial}$ ) cases suggest that it may be possible to replace the condition $|u(x)| \leq \varepsilon(\|x\|)$ by a requirment that $u$ should decay rapidly, but not a priori uniformly, on a suitable set of unbounded paths; see, for example, Armitage and Goldstein [2]. Here we confirm that there is indeed a general result of this type.

We now fix some further notation. Let $M$ be an $(n-1)$-dimensional manifold, and let $\Gamma: M \times[0,+\infty) \rightarrow \Omega$ be a continuous function such that (i) $\Gamma(\omega \times(0,+\infty))$ is open for each open subset $\omega$ of $M$, and (ii) for each $\xi \in M$ the set $\gamma_{\xi}=\{\Gamma(\xi, t): t \geq 0\}$ is closed and unbounded.

Theorem 1. There exists a continuous function $\eta:[0,+\infty) \rightarrow(0,1]$ with the following property. If $u$ is a solution of $P(D) u=0$ on $\Omega$ such that

$$
u(x)=O(\eta(\|x\|)) \quad\left(\|x\| \rightarrow+\infty, x \in \gamma_{\xi}\right)
$$

for a second category set of $\xi$ in $M$, then $u \equiv 0$.

Received by the editors November 22, 1993.

1991 Mathematics Subject Classification. Primary 35J30. 
The category condition is indispensible, at least in the holomorphic case: if $S$ is a first category subset of the unit circle and $\eta:[0,+\infty) \rightarrow(0,1]$ is continuous, then there exists a non-constant entire function $f$ such that $f(r z)=o(\eta(r))$ as $r \rightarrow+\infty$ for each $z \in S$ (see Schneider [4, Example 10]).

In proving Theorem 1 , we indicate first how $\eta$ is chosen. Let $\left\{B_{1}, B_{2}, \ldots\right\}$ be a countable base for the topology of $M$. For the moment let $k$ be a fixed positive integer. By hypothesis, $\Gamma\left(B_{k} \times(0,+\infty)\right)$ is open. Moreover, this set has an unbounded connected component, since it contains an unbounded connected set of the form $\Gamma(\{\xi\} \times(0,+\infty))$. Let $\Omega_{k}$ be an unbounded, connected, open subset of $\Gamma\left(B_{k} \times(0,+\infty)\right)$ such that $\bar{\Omega}_{k} \subset \Omega$. By Theorem A, there exists a continuous function $\varepsilon_{k}:[0,+\infty) \rightarrow(0,1]$ with the property that the zero function is the only solution of $P(D) u=0$ on $\Omega_{k}$ satisfying $|u(x)| \leq \varepsilon_{k}(\|x\|)$ for all $x \in \Omega_{k}$. We take $\eta:[0,+\infty) \rightarrow(0,1]$ to be a continuous function such that $\eta \leq \varepsilon_{k}$ on $(k,+\infty)$ for each $k$.

Now suppose that $u$ is a solution of $P(D) u=0$ on $\Omega$ satisfying (1) for all $\xi$ belonging to a second category subset $E$ of $M$, and define a function $\Phi$ on $M$ by

$$
\Phi(\xi)=\sup \left\{|u(x)| / \eta(\|x\|): x \in \gamma_{\xi}\right\} .
$$

We claim that $\Phi$ is lower semi-continuous on $M$. To prove this, suppose that $\xi \in M$ and that $A<\Phi(\xi)$. Then there exists $x \in \gamma_{\xi}$, say $x=\Gamma(\xi, t)$, such that $|u(x)| / \eta(\|x\|)>A$. By the continuity of $u, \eta$ and $\Gamma$, there exists $\delta>0$ such that $|u(y)| / \eta(\|y\|)>A$ whenever $\|x-y\|<\delta$, and there exists an open neighbourhood $N$ of $\xi$ such that $\|x-\Gamma(\zeta, t)\|<\delta$ for all $\zeta \in N$. Hence

$$
\Phi(\zeta) \geq|u(\Gamma(\zeta, t))| / \eta(\|\Gamma(\zeta, t)\|)>A \quad(\zeta \in N),
$$

so $\Phi$ is lower semi-continuous at $\zeta$. Now define $A_{m}=\{\xi \in M: \Phi(\xi) \leq$ $m\}(m=1,2, \ldots)$. By the lower semi-continuity of $\Phi$, each $A_{m}$ is closed. Clearly $\Phi(\xi)<+\infty$ for each $\xi \in E$, so that $E \subseteq \bigcup_{m=1}^{\infty} A_{m}$. Since $E$ is second category, some $A_{k}$ has non-empty interior; this interior contains some $B_{q}$. If $x \in \Omega_{q}$, then $x \in \gamma_{\xi}$ for some $\xi \in B_{q} \subseteq A_{k}$, so that $|u(x)| / \eta(\|x\|) \leq \Phi(\xi) \leq k$. Hence

$$
|u(x)| \leq k \eta(\|x\|) \leq k \varepsilon_{q}(\|x\|) \quad\left(x \in \Omega_{q},\|x\|>q\right) .
$$

Since, further, $u$ is bounded on the compact set $\left\{x \in \bar{\Omega}_{q}:\|x\| \leq q\right\}$, there exists a positive constant $C$ such that $C|u(x)| \leq \varepsilon_{q}(\|x\|)$ for all $x \in \Omega_{q}$. From our choice of $\varepsilon_{q}$, it follows that $u=0$ on $\Omega_{q}$. Since $u$ is real-analytic on $\Omega$, we conclude that $u=0$ on $\Omega$.

\section{REFERENCES}

1. D. H. Armitage, T. Bagby, and P. M. Gauthier, Note on the decay of solutions of elliptic equations, Bull. London Math. Soc. 17 (1985), 554-556.

2. D. H. Armitage and M. Goldstein, Radial limiting behaviour of harmonic functions in cones, Complex Variables 22 (1993), 267-276.

3. K. F. Barth, D. A. Brannan, and W. K. Hayman, Research problems in complex analysis, Bull. London Math. Soc. 16 (1984), 490-517.

4. W. J. Schneider, Approximation and harmonic measure, Aspects of Contemporary Complex Analysis (D. A. Brannan and J. G. Clunie, eds.), Academic Press, London, 1980, pp. 333-349.

Department of Pure Mathematics, Queen's University, Belfast BT7 INN, Northern IRELAND

E-mail address: d.armitage@uk.ac.qub.v2 